\title{
FUNCTIONS OF FINITE $\lambda$-TYPE IN SEVERAL COMPLEX VARIABLES
}

\author{
BY ROBERT O. KUJALA ${ }^{1}$ \\ Communicated by I. Glicksberg, September 26, 1968
}

In [1] L. A. Rubel and B. A. Taylor present some results in the theory of meromorphic and entire functions of finite $\lambda$-type on the plane. By applying Fourier series techniques they are able to achieve a generalization of a classical theorem of Lindelöf concerning the existence of an entire function of restricted order and type having a prescribed set of zeros. In [2] Wilhelm Stoll develops a generalization of this theorem of Lindelof for the case of functions of several variables using the techniques of value distribution theory. The purpose of this note is to report some results toward a comprehensive theory of functions of finite $\lambda$-type in several complex variables which are produced by combining the techniques of Rubel and Taylor with those of Stoll.

A continuous nondecreasing function from the positive real numbers into the positive real numbers is called a growth function. If $\lambda$ is a growth function, then a meromorphic function $f$ on $C^{k}$ is said to be of finite $\lambda$-type if the upper limit at infinity of the quotient $T(r, s ; f) / \lambda(B r)$ is finite for some positive constants $s$ and $B$, where $T(r, s ; f)$ is the (Kneser-Stoll) characteristic of $f$ for $r \geqq s \geqq 0$. If, in particular, $f$ is entire, then $f$ is of finite $\lambda$-type if and only if there are positive constants $A, B$, and $R$ such that $|f(\zeta)| \leqq \exp (A \lambda(B|\zeta|))$ for all $\zeta$ in $C^{k}$ with $|\zeta|>R$.

The class of all meromorphic functions on $C^{k}$ which are of finite $\lambda$-type for a given $\lambda$ is a field under the usual operations on functions. Moreover, this field is invariant under parallel translations of the variable in $\boldsymbol{C}^{k}$ and is either the class of constant functions or contains all rational functions on $\boldsymbol{C}^{k}$. Similarly, the class of all entire functions on $C^{k}$ which are of finite $\lambda$-type for a given $\lambda$ is an integral domain. These facts are immediate consequences of the properties of the characteristic. A natural question, then, is the following: For which growth functions $\lambda$ is the field of meromorphic functions of finite

${ }^{1}$ An abstract of a dissertation written under the direction of Professor Wilhelm Stoll and submitted to the Graduate School of the University of Notre Dame in partial fulfillment of the requirements for the degree of Doctor of Philosophy. This research was partially supported by the National Science Foundation under grants numbered GP-3988 and GP-7265. 
$\lambda$-type on $C^{k}$ precisely the field of quotients of the integral domain of all entire functions of finite $\lambda$-type on $C^{k}$ ? A growth function which has the desired quotient property for functions on $C^{k}$ will be called regular for $k$. A necessary and sufficient condition that a growth function be regular for 1 is provided by Rubel and Taylor in [1]. The results of this note give a sufficient condition for regularity in the higher dimensional cases.

If $f$ is a function which is holomorphic and not identically zero on an open connected subset $U$ of $C^{k}$, then the divisor of $f, \nu_{f}$, is that integer-valued function on $U$ whose value at each point is the zeromultiplicity of $f$ at that point. If $f$ is a function which is meromorphic and not identically zero on $C^{k}$, then for each point $\zeta$ of $C^{k}$ there exists an open connected neighborhood $U$ of $\zeta$ and two functions $g$ and $h$ which are holomorphic and not identically zero on $U$ such that $h f=g$ on $U$ and $g$ and $h$ are relatively prime at $\zeta$. The function $\nu_{f}^{0}$ taking $\zeta$ to $\nu_{g}(\zeta)$ is called the zero-divisor of $f$ and $\nu_{j}^{\infty}$ taking $\zeta$ to $\nu_{h}(\zeta)$ is called the pole-divisor of $f$. The divisor of $f, \nu_{f}$, is $\nu_{f}^{0}-\nu_{f}^{\infty}$. It follows that the divisor of a product of functions is the sum of the divisors of the factors, the divisor of the reciprocal of a function is the negative of the divisor of the function, and a meromorphic function on $C^{k}$ is entire if and only if its divisor is nonnegative, that is, if and only if its divisor and its zero-divisor coincide. Finally, a divisor on $\boldsymbol{C}^{k}$ is any integer-valued function on $C^{k}$ which is locally the divisor of a meromorphic function.

If $f$ is a meromorphic function on $\boldsymbol{C}^{k}$ which is holomorphic in a neighborhood of the origin, then the restriction of $f$ to $\zeta$ in $C^{k}$ is that meromorphic function on $\boldsymbol{C}$ defined by $f \mid \zeta(z)=f(z \zeta)$. If $\nu$ is a nonnegative divisor on $C^{k}$ with $\nu(0,0, \cdots, 0)=0$, then, since $C^{k}$ is a Cousin II domain, there is an entire function $f$ on $C^{k}$ such that $\nu_{f}=\nu$ and $f(0,0, \cdots, 0)=1$; thus, the restriction of $\nu$ to $\zeta$ in $C^{k}$ may be defined by $\nu \mid \zeta=\nu_{f \mid \zeta}$. The growth of such a divisor is measured by the following indices for $r>s \geqq 0, \zeta$ in $C^{k}$, and $p$ a natural number:

$$
\begin{aligned}
n(r ; \nu, \zeta) & =\sum_{|z| \leq r} \nu \mid \zeta(z), \\
N(r ; \nu, \zeta) & =\int_{0}^{r}(1 / t) n(t ; \nu, \zeta) d t, \\
\sum_{p}(r, s ; \nu, \zeta) & =\frac{1}{p} \sum_{<<|\Delta| \mathbf{s} r}[\nu \mid \zeta(z)] z^{-p} .
\end{aligned}
$$

Definitron. A nonnegative divisor $\nu$ on $C^{k}$ with $\nu(0,0, \cdots, 0)=0$ is said to be of finite $\lambda$-density if and only if there exist positive constants $A, B$ and $R$ such that 


$$
N(r ; \nu, \zeta) \leqq A \lambda(B r)
$$

for all $r>R$ and all unit vectors $\zeta$ in $C^{k}$. If, in addition, $A, B$ and $R$ may be chosen so that

$$
\left|\sum_{p}(r, s ; \nu, \zeta)\right| \leqq r^{-p} A \lambda(B r)+s^{-p} A \lambda(B s)
$$

for all natural numbers $p$, all $r>s>R$, and all unit vectors $\zeta$ in $C^{k}$, then $\nu$ is said to be $\lambda$-admissible.

Definition. A growth function $\lambda$ is said to be strictly regular for $k$ if and only if for every divisor $\nu$ which is of finite $\lambda$-density on $C^{k}$ there is a $\lambda$-admissible divisor $\nu^{\prime}$ on $C^{k}$ such that $\nu^{\prime} \geqq \nu$.

The main result may now be stated as follows:

THEOREM. If $\lambda$ is strictly regular for $k$, then $\lambda$ is regular for $k$. The converse is also true when $k=1$.

As consequences of this theorem we have

Proposition. If $\lambda$ is "slowly growing," i.e., if there is a constant $B>1$ such that $\lambda(B r) / \lambda(r)$ is bounded for $r$ sufficiently large, then $\lambda$ is regular for all dimensions.

Moreover, since $\log r$ and $r^{p}$ are slowly growing functions of $r$ for $\rho>0$ it follows that:

Proposition. Let $\rho \geqq 0$ be given. Then every meromorphic function on $C^{k}$ which is of order less than $\rho$ or of order $\rho$ and finite type is the quotient of two entire functions on $C^{k}$ each of which is of order less than $\rho$ or of order $\rho$ and finite type.

The proof of the theorem is contained in the following two results:

Proposition. If a nonnegative divisor $\nu$ on $C^{k}$ with $\nu(0,0, \cdots, 0)=0$ is the pole divisor of some meromorphic function of finite $\lambda$-type on $C^{k}$, then $\nu$ is of finite $\lambda$-density.

Proposition. A nonnegative divisor $\nu$ on $C^{k}$ with $\nu(0,0, \cdots, 0)$ $=0$ is the divisor of an entire function of finite $\lambda$-type on $C^{k}$ with $f(0,0, \cdots, 0)=1$ if and only if $\nu$ is $\lambda$-admissible.

The proof of the first of these propositions involves the demonstration of the fact that for any meromorphic function $f$ on $C^{k}$ which is holomorphic in a neighborhood of the origin there are positive constants $A, B, C$ and $R$ such that

$$
T(r, 0 ; f \mid \zeta) \leqq A T(B r, 0 ; f)+C
$$


for all $r>R$ and all unit vectors $\zeta$ in $C^{k}$. Following this the result follows from an application of the techniques of Rubel and Taylor for functions of one variable. The necessity of $\lambda$-admissibility in the second proposition is deduced in a similar manner. Finally, the sufficiency of $\lambda$-admissibility can be shown by a careful modification of Fourier series techniques and the techniques of Stoll.

It is possible to construct an analogous theory of functions of zero $\lambda$-type by replacing "finite" with "zero" and by substituting a function " $A(r)$," which is positive, continuous and decreasing to zero at infinity, for the constant " $A$ " in the preceding definitions and theorems. Then every slowly growing function is also zero regular for all dimensions.

It is interesting to note that if a growth function is regular (or zero regular) for some dimension, then it is regular (or zero regular) for all lower dimensions. And it is tempting to conjecture that all growth functions are regular and zero regular.

A growth function $\lambda$ is called extraregular for $k$ provided that every meromorphic function of finite $\lambda$-type on $C^{k}$ is the quotient of two entire functions of finite $\lambda$-type on $\boldsymbol{C}^{k}$ which are locally relatively prime at every point. And $\lambda$ is strictly extraregular for $k$ if every divisor of finite $\lambda$-density on $C^{k}$ is $\lambda$-admissible. Then every strictly extraregular growth function is extraregular. The functions $\lambda(r)=r^{p}$ for positive nonintegral numbers $\rho$ are extraregular for all dimensions. In the analogous situation, the functions $\lambda(r)=r^{\rho}$ for $0<\rho<1$ are zero extraregular for all dimensions.

\section{REFERENCES}

1. L. A. Rubel and B. A. Taylor, $A$ Fourier series method for meromorphic and entire functions, Bull. Amer. Math. Soc. 72 (1966), 858-860; Bull. Soc. Math. France 96 (1968), 53-96.

2. Wilhelm Stoll, About entire and meromorphic functions of exponential type, Proc. Sympos. Pure Math., vol. 11, Amer. Math. Soc., Providence, R. I., 1968, pp. 392-430.

Tulane University, New Orleans, Louisiana 70118 